\title{
Quality of Life in Patients with Dementia with Lewy Bodies
}

\author{
Chuo-Yu Lee, ${ }^{1,2,3}$ Shih-Jung Cheng, ${ }^{1,3,4}$ Hui-Chi Lin, ${ }^{1}$ Yu-Lu Liao, ${ }^{5}$ and Pei-Hao Chen $\mathbb{D}^{1,3,6}$ \\ ${ }^{1}$ Department of Neurology, Mackay Memorial Hospital, Taipei, Taiwan \\ ${ }^{2}$ Graduate Institute of Chemistry, Tamkang University, New Taipei City, Taiwan \\ ${ }^{3}$ Department of Medicine, Mackay Medical College, New Taipei City, Taiwan \\ ${ }^{4}$ Department of Physical Therapy and Assistive Technology, National Yang-Ming University, Taipei, Taiwan \\ ${ }^{5}$ Department of Accounting Information, Takming University of Science and Technology, Taipei, Taiwan \\ ${ }^{6}$ Graduate Institute of Mechanical and Electrical Engineering, National Taipei University of Technology, Taipei, Taiwan
}

Correspondence should be addressed to Pei-Hao Chen; a7662888@gmail.com

Received 1 February 2018; Accepted 6 June 2018; Published 5 July 2018

Academic Editor: Ying-Jui Ho

Copyright (C) 2018 Chuo-Yu Lee et al. This is an open access article distributed under the Creative Commons Attribution License, which permits unrestricted use, distribution, and reproduction in any medium, provided the original work is properly cited.

\begin{abstract}
Dementia with Lewy bodies (DLB) is a complex, multisymptom disorder. When making decisions regarding the treatment of DLB, the patient's quality of life (QoL) should always be the main consideration. To our knowledge, this is the first review article focusing on the QoL in DLB patients. We searched the PubMed database using the keywords "quality of life" and "dementia with Lewy bodies." Previously, no specific instrument had been developed for assessing the QoL in DLB patients. Patients with DLB have a decreased QoL compared to patients with Alzheimer's disease, which is reportedly caused by several factors including level of independence in instrumental activities of daily living, whether the patient is living with the caregiver, apathy, delusion, and dysautonomia. The direct effect of visual hallucination, sleep, and movement disorders on the QoL in DLB patients has not been previously studied. The role of cognitive function on the QoL is still controversial. In a randomized controlled study, memantine may improve the QoL in PDD or DLB patients. We concluded that it is important to develop a specific instrument to assess the QoL in DLB patients. Furthermore, there is an urgent need for large clinical trials to identify factors associated with the QoL and how they can be managed.
\end{abstract}

\section{Introduction}

Dementia with Lewy bodies (DLB) is the second most common neurodegenerative dementia after Alzheimer's disease $(\mathrm{AD})$ in people over 65 years of age. A recent published population-based cohort study from the Taiwan National Health Insurance Research Database showed the incidence of DLB was 7.10 per 100,000 person-years [1]. DLB differs from $\mathrm{AD}$ as early cognitive symptoms include deficits in visuospatial and executive function, rather than memory. DLB is characterized by parkinsonism, fluctuations in mental status, visual hallucination, and hypersensitivity to neuroleptics. Therefore, it is difficult to discriminate between DLB and Parkinson's disease with dementia (PDD). Both diseases also share a similar pathologic finding, Lewy bodies. Generally, if cognitive impairments appear within a year of parkinsonism,
DLB is diagnosed, while patients with parkinsonism for at least a year prior to cognitive impairment are classified as PDD. Early amyloid deposition in DLB relative to PDD may explain the difference in the timing of dementia and parkinsonism [2].

The quality of life (QoL) is a key outcome measure of health and social service interventions. Currently, patientreported outcome measures are increasingly used in evaluating health and social care. Definitions of health-related QoL include physical, mental, social, and role functioning and health perceptions [3]. Because aging is a global issue, it is critical to identify QoL determinants in the elderly who suffer from chronic disease. A recent study from Southern Taiwan showed Alzheimer disease-8, a screening tool, had the strongest association with the total QoL score in 115 old-age adults suffering from chronic disease. It is important for geriatric 
health care providers to realize that cognitive impairment among old age adults with chronic disease is a critical determining factor of poor QoL [4].

Assessing the QoL in people with dementia is crucial for evaluating their outcomes; however, it is challenging to interview patients who have a limited ability to express themselves and may lack insight. Meanwhile, there are questions concerning the validity of generic measures of QoL, which are not specific to dementia. Currently, evaluations of QoL largely focus on general dementia or AD. A systematic review [5] regarding the QoL in dementia strongly suggests that depression is consistently related to a decreased QoL. Surprisingly, there is no convincing evidence indicating that lower cognitive ability or greater limitations in the activity are associated with a lower QoL.

Evidence concerning the QoL in DLB patients is limited [6]. In a comparison study, the QoL was measured using the Alzheimer Disease-Related Quality of Life (ADRQL) evaluation, a proxy-rated, dementia-specific instrument. The authors concluded that DLB patients have a poorer QoL than AD patients [7]. Figari et al. compared the QoL in 46 AD, 23 DLB, and 39 Huntington's disease (HD) patients, all of whom had dementia for at least two years. Patients with DLB scored significantly lower on the SF-12 Physical and Mental Health Summary than patients with HD and AD. The authors concluded that, when adjusted for age, cognition, comorbidity, and depression, patients with DLB had the poorest QoL [8].

There is only one study whose primary aim was to compare the QoL in patients with DLB and AD [9]. 34 DLB patients and 34 cognitive-matched $\mathrm{AD}$ patients were evaluated using two QoL instruments: EQ-5D and QoL-AD. The results showed both patient-rated QoL and proxy-rated QoL were poorer in patients with DLB than those with AD. Due to many differences in clinical symptoms, it is reasonable to predict differences in the QOL between diseases. Herein, we elaborate on studies related to the QoL in DLB patients.

\section{Methods}

Studies were selected from the PubMed database using keywords "quality of life" and "dementia with Lewy bodies." All languages (with an English abstract) were included. Articles spanned the period between June 1986 and December 2017. Searches yielded 102 citations of which 21 were included as relevant. Other data were collected through references from selected articles and searching relevant journals offline.

2.1. Dementia-Specific QoL Measurement Scales. Unfortunately, there are no instruments available to specifically evaluate the QoL in DLB patients. Therefore, we herein discuss QOL measurement scales for dementia.

There is some debate regarding whether to use the patient's or caregiver's reported QoL as the measurement. Typically, the QoL is rated higher by patients than by caregiver proxy measures. The differences are associated with increased levels of caregiver burden and caregiver depression, rather than lower levels of patients' cognitive performance [10]. Therefore, it is reasonable to directly assess subjective QoL in patients with mild to moderate dementia. Interestingly, Karlawish et al. proposed a method to minimize the discrepancy between patient's and caregiver's QoL scores: caregivers should rate using substituted judgment as if they were the patients [11]. Issues regarding the exclusion of patients with severe dementia from selfreported questionnaires and weak reliability of proxy versions remain unresolved.

There is limited knowledge regarding the standard instruments used to evaluate the QoL in dementia patients. In a recently published review [12], the most frequently used dementia-specific instrument was the Quality of Life in Alzheimer's Disease (QOL-AD) and Dementia Quality of Life questionnaire (DEMQOL). As for generic measures, EuroQol 5-dimension (EQ-5D) and Short Form surveys (SF-36 or SF-12) were widely used. The authors recommended dementia-specific DEMQOL, generic SF-12, and health utility EQ-5D-5L, based on both self- and proxy-report [12]. In the following paragraphs, we will briefly introduce each widely-used measurement scale.

2.2. Quality of Life in Alzheimer's Disease (QOL-AD). The QOL-AD [13] is an instrument specifically developed for mild to severe dementia. It includes 13 items, including physical health, energy, mood, living situation, memory, family, marriage, friends, self, ability to do chores, ability to do things for fun, money, and life as a whole. Each domain is rated from 1 (poor) to 4 (excellent). As for the caregiver version, which contains 15 items, marriage and money are removed, while people who work here, ability to take care of oneself, ability to live with others, and ability to make choices in one's life are added.

2.3. Dementia Quality of Life Questionnaire (DEMQOL). The DEMQOL [14] aims to assess QoL in mild to moderate dementia. It includes 5 domains including daily activities and looking after self, health and well-being, cognitive functioning, social relationships, self-concept, and a total of 28 items. Each item is scored from 1 to 4 . A proxy version has been developed for caregivers, DEMQOL-Proxy, which contains 31 items.

2.4. EuroQol 5-Dimension (EQ-5D-5L). The EQ-5D-5L [15] is a generic instrument. Respondents are asked to rate their current health status on 5 dimensions: mobility, hygiene, usual activities, pain/discomfort, and anxiety/depression. For each dimension, the respondent gives 1 of 5 possible ratings according to symptom severity. The questionnaire also includes a visual analog scale (VAS) as a secondary part, ranging from 0 (death) to 100 (perfect health). Thus, two QoL values are acquired with this instrument.

2.5. Short Form-36 and Short Form-12 (SF-36 and SF-12). The SF-36 [16] is a generic measurement containing 36 questions across 8 dimensions of health status: vitality, physical functioning, bodily pain, general health perceptions, physical role functioning, emotional role functioning, social role functioning, and mental health. A shorter version, SF-12, was 
TABLE 1: Characteristics of QoL measurement scales for dementia.

\begin{tabular}{|c|c|c|c|c|}
\hline Instrument & $\begin{array}{l}\text { Patient } \\
\text { report }\end{array}$ & $\begin{array}{l}\text { Proxy } \\
\text { report }\end{array}$ & Domains & Items \\
\hline $\begin{array}{l}\text { Quality of Life } \\
\text { in Alzheimer's } \\
\text { Disease } \\
\text { (QOL-AD) }\end{array}$ & Yes & Yes & $\begin{array}{c}\text { Physical health } \\
\text { Mental health } \\
\text { Social and functional } \\
\text { domains }\end{array}$ & 13 (15 for proxy version) \\
\hline
\end{tabular}

Measurement

Four-point scale score Range 13-52 for patient report and range $15-60$ for proxy report Higher scores equal to higher QoL

Four-point scale score Daily activities and looking after self

Dementia

Quality of Life questionnaire

Yes Yes (DEMQOL)

Health and well-being

Cognitive functioning Social relationships

28 (31 for proxy version) Range 28-112 for patient for proxy report

Higher scores equal to Self-concept report and range 31-124 higher QoL

1st part: having no

1st part:

Mobility

Hygiene

EuroQol

5-dimension

Yes

No

(EQ-5D-5L)

Usual activities

Pain/discomfort

Anxiety/depression 2nd part:

Visual analogue scale (VAS)

Vitality

Physical functioning

Bodily pain

Short Form-36

(SF-36)

Yes No

slight problems for 2 , having moderate

problems for 3 , having severe problems for 4 , and being unable to

do/having extreme problems for 5

2nd part: 0 for worst; 100 for best health status

One score in each special software, representing weighted sums of the questions

( 0 equivalent to maximum disability;

100 equivalent to no disability) problems for 1, having domain, calculated by
General health perceptions

cal ro functioning

Emotional role functioning

Social role functioning

Mental health
Brief summary

The best researched of all the dementia

measurement scales Relatively brief

Suitable for mild to severe dementia

The conceptual

framework is based

on health-related

QoL, with less

social relevance

The proxy version

performed well in

psychometric tests

Suitable for mild to

moderate dementia

A generic, preference-based

instrument for health related QoL

The 5-digit number

for 5 domains can be

converted into a

single weighted

index score

Appropriate in

people with mild to

moderate dementia

A generic health

status measure

A shorter version,

SF-12, was developed

to minimize

respondent burden if

only physical and

mental health

summary scores are of interest developed to minimize respondent burden if only physical and mental health summary scores are of interest.

A summary of the four widely-used scales is provided in Table 1.

2.6. Reported Symptoms That May Affect QoL in DLB Patients. One study compared the QoL in patients with $\mathrm{DLB}$ and $\mathrm{AD}$ as its primary aim and demonstrated that the following are important determinants of the QoL in DLB patients: their Neuropsychiatric Inventory score and level of independency in instrumental activities of daily living (ADL), whether the patient lives with the caregiver, apathy, and delusion [9]. Similar to most findings in overall dementia, cognitive function has no strong relation to the QoL in this study.

Caregivers play an important role in affecting a patient's QoL. In a cross-sectional study [17] of 161 patients with dementia (including 13 DLB patients), the QoL was measured using the ADRQL. The results indicated that some predictors of a reduced QoL include behavioral and depressive symptoms, dependency in basic ADL, poorer cognitive function, use of antipsychotic medication, caregiver burden, and caregiver not being an adult child. The role of cognitive function on the QoL is still controversial. Here, we will discuss some reported causative factors for a poorer QoL in DLB patients.

2.7. Visual Hallucinations. Visual hallucinations occur in 60 to $70 \%$ of DLB patients, usually beginning in the first 2 or 3 years of the disease [18]. The presentation of visual hallucinations in the first 4 years following the onset of dementia has a positive and negative predictive value for DLB of $81 \%$ and $79 \%$, respectively [19]. The most common visual hallucinations are fully formed persons (84\%), animals or bugs (37\%), and objects (39\%) [20]. Early misperceptions and misidentification of family members are usually reported in 
DLB. Capgras syndrome, a delusion where a person has been substituted by an imposter with a similar outward, is also common in DLB and is associated with higher rates of visual hallucinations and anxiety [21]. A recently published postmortem study [22] showed DLB cases had reduced neuronal density in the intermediate gray layer of the superior colliculus tissue, a structure important for directing attention toward visual targets. This finding may provide pathologic evidence for visual hallucinations in DLB.

A cross-sectional and retrospective study of 1025 patients with dementia in Spain found that delusion and hallucinations were more prevalent in DLB patients than those with $\mathrm{AD}$ and PDD [23]. In a recent comparative study of 207 delusional and nondelusional patients with DLB, the authors concluded that the delusional patients had poorer cognitive function and more severe neuropsychiatric symptoms [24]. In a cross-sectional study, $21 \mathrm{DLB}$ and $35 \mathrm{PDD}$ patients with recurrent visual hallucinations were evaluated. Most patients had complex hallucinations daily, usually lasting minutes. The study showed that neuropsychiatric symptoms that coexist with hallucinations are apathy, sleep disturbance, and anxiety [25]. The direct effect of visual hallucination on the QoL in DLB patients has not been previously studied.

Treatment of psychosis is challenging in DLB, since many patients are hypersensitive to neuroleptic drugs; therefore, nonpharmacologic treatment approaches should be considered first. Typical antipsychotic agents should be avoided, while the evidence for atypical antipsychotics is controversial. At present, quetiapine and clozapine are usually prescribed. Pimavanserin, a selective serotonin 5HT2A inverse agonist, has beneficial effects on treating psychosis in PDD [26], while similar results are expected in DLB patients.

2.8. Depression and Apathy. The presence of depression and other behavioral and psychological symptoms of dementia (BPSD) may worsen the QoL of dementia patients and their caregivers [27]. The BPSD can be classified into four clusters: depression, aggressive behaviors, psychosis, and euphoria. In $\mathrm{AD}$ and DLB patients, a cross-sectional analysis determined that depressive symptom in patients may be the most important BPSD symptom as it was the only one shown to cause depression in caregivers [28].

A previous literature revealed that DLB is associated with higher scores on the Geriatric Depression Scale compared to $\mathrm{AD}$, and a higher rate of depression is found in the early stages of the disorder [29]. Kurisu et al. compared the QoL of 279 degenerative dementia patients with different subtypes by using the QOL Questionnaire for Dementia (QOL-D) as an evaluation scale. The QOL-D comprises six domains: positive affect, negative affect and actions, communication, restlessness, attachment to others, and spontaneity. Results showed that apathy in frontotemporal dementia (FTD) and DLB patients and depression in DLB patients might cause the reduced positive affect in FTD and DLB patients compared to $\mathrm{AD}$ patients [30]. Apathy also plays an important role in evaluating the QoL in DLB patients. In a DLB and $\mathrm{AD}$ comparison study, DLB patients are less able to complete the QoL questionnaire [6].
In DLB, the presence of Lewy bodies in limbic, paralimbic, and neocortical regions may account for the appearance of depressive symptoms [27]. Early detection of depression in DLB patients is important as these symptoms are treatable. In a recently published review, the authors concluded that neuropsychiatric symptoms, especially psychosis and depression, are priority targets for intervention to improve the outcome of patients with DLB [6].

2.9. Sleep Disorders. Sleep disorders are common in DLB patients, with both rapid eye movement sleep behavior disorder (RBD) and fluctuating cognition being part of the clinical diagnostic criteria. In a contemporary review in sleep medicine, the authors concluded that appropriate management of sleep-related symptoms can improve the QoL in patients with neurodegenerative disorders [31]. Orexins (also known as hypocretins) are secreted in neurons in the lateral hypothalamus that are related to sleep regulation and attention [32]. Lower levels of orexin-1 were detected in the cerebrospinal fluid of DLB patients compared to AD patients and control subjects [33].

$76 \%$ of DLB patients had RBD [34], which is characterized by acting out dreams, resulting in vocalizations and even violent behavior. Other nocturnal symptoms such as anxiety, periodic leg movements, urinary dysfunction, and difficulty turning over in bed can contribute to sleep problems [31]. In a retrospective study of 78 patients with DLB and sleep disorders who underwent polysomnography, approximately three quarters experienced many arousals not accounted for by a movement or breathing disturbance. Among patients who did not show evidence of significantly disordered breathing, $62 \%$ of arousals were arousals for no apparent reason [35]. The direct effect of sleep disorders on the QoL in DLB patients has not been previously studied.

2.10. Dysautonomia. In a study [36] of the prevalence of autonomic symptoms in dementia, the authors concluded that total autonomic symptom scores [37], urinary symptoms, constipation, and postural dizziness were significantly higher in DLB patients than in AD patients. By using the SF-36 [38] as a measurement, higher autonomic symptom scores were related to a lower QoL. The authors proposed that the effect of autonomic symptoms upon the QoL may be due to the limitations in ADL.

A systematic review of autonomic dysfunction in $\alpha$-synucleinopathies revealed that cardiovascular autonomic failure has a significant impact on daily activities and QoL. Cerebral white matter changes in image and cognitive decline may be related to altered cerebral perfusion, vascular pressure stress, and associated disruption of the blood-brain barrier [39]. In $\alpha$-synucleinopathies, autonomic dysfunction is more severe in DLB than in PD patients [40].

Both preganglionic and postganglionic dysfunctions may be present in DLB patients [41]. In a retrospective examination of DLB patients, urinary incontinence and constipation were the most commonly presented autonomic symptoms, occurring in 97 and $83 \%$ of patients, respectively, while syncope occurred in $28 \%$ of patients [42]. Orthostatic hypotension $(\mathrm{OH})$ has been reported in around $50 \%$ of DLB 
patients [43]. OH is defined as the reduction in systolic blood pressure of at least $20 \mathrm{mmHg}$ or diastolic blood pressure of $10 \mathrm{mmHg}$ within 3 minutes of standing from the lying position [44]. Patients may not present with classic postural dizziness, but instead nonspecific malaise or lethargy, which can markedly increase the risk of falls or syncope. Management may include salt supplementation, compression stockings, or oral medication such as midodrine or fludrocortisone [45]. Furthermore, a higher prevalence of carotid sinus syndrome, an altered arterial sinus response to baroceptive stimulation resulting in syncope, was found in $32 \%$ of DLB patients compared to $11.1 \%$ of AD patients [46].

\subsection{Reported Management That May Improve QoL in DLB} Patients. It can be helpful to divide the symptoms of DLB into five categories: cognitive, neuropsychiatric, movement, autonomic, and sleep. It is important to take a detailed history and form a comprehensive treatment strategy to improve a patient's QoL [47]. There is little published data regarding the effect of the management of symptoms on the QoL in DLB patients.

2.12. Armodafinil. Because armodafinil has a longer half-life than modafinil, we may predict its better effect at treating patients with excessive daytime sleepiness [48]. In a 12week pilot trial of oral armodafinil therapy $(120-250 \mathrm{mg}$ daily), DLB patients with hypersomnia showed improved scores on the Epworth Sleepiness Scale, Maintenance of Wakefulness Test, and Clinical Global Impression of Change. Moreover, caregivers' overall QoL improved at week 12 [49]. It should be noted that the effect of armodafinil on patients' QoL was not analyzed in this trial.

2.13. Memantine. Memantine, an NMDA receptor antagonist, has been widely used in treating moderate to severe $\mathrm{AD}$. A randomized controlled study was conducted on 70 patients with PDD or DLB over 24 weeks using caregiverrated QOL-AD as a measurement. A secondary analysis of this study showed that memantine improved the total QoL, body function, physical health, energy, mood, and memory when compared to the placebo group [50]. Large-scale trials are required to confirm these preliminary findings. On the other hand, although cholinesterase inhibitors are widely prescribed for patients with DLB, we found no reported effect of cholinesterase inhibitors on the QoL in DLB patients.

2.14. Exercise. Exercise may improve functional outcomes in $\mathrm{PD}$ and $\mathrm{AD}$ patients. The multisymptom nature of the DLB (parkinsonism, cognitive, and psychiatric) results in these patients often being excluded from clinical trials to avoid confounding results. A recent systematic review confirms a scarcity of research investigating exercise as a potential therapy in DLB patients [51]. The authors concluded that the effect of exercise on cognitive, psychiatric, QoL, and physiological outcomes remains unclear in DLB patients. Further clinical trials in larger cohorts are necessary.

\section{Conclusions}

This is, to our knowledge, the first review article focusing on the QoL in patients with dementia with Lewy bodies. Our review may not be fully comprehensive due to the limited literature available regarding this topic. Physicians should keep the patient's QoL in the forefront when managing their symptoms. The following are reported factors leading to poorer QoL in DLB patients: independence in instrumental $\mathrm{ADL}$, whether the patient is living with the caregiver, the presence of depression, apathy, delusion, and dysautonomia.

First, we concluded that it is important to develop a specific instrument to assess the QoL in DLB patients. Apathy is more common in DLB patients, causing them less able to complete the questionnaire [6]. Therefore, it is reasonable to design a scale with proxy version. Since DLB is a complex, multisymptom disorder, we recommend the following domains should be taken into account: health and well-being, daily activities, cognitive functioning, positive and negative affect, restless behavior, social interaction, and satisfaction (i.e., restful sleep). Second, we hope that with the advent of more diagnostic criteria and advances in biomarkers, there will be well-defined DLB cohorts for clinical trials. Further studies may be facilitated by international or multicenter cooperation. For example, a large longitudinal cohort of 5624 DLB patients has been developed recently in 135 dementia centers in Italy (the DLB-SINdem study group) [52]. There is an urgent need for large, cross-sectional, and longitudinal trials to determine other factors associated with the QoL, such as cognition, visual hallucination, sleep, and movement disorders. Finally, because of the poor prognosis and high socioeconomic burden of DBL, we should consider the health-related QoL as one of the outcome measurements when developing new drugs for DLB.

\section{Conflicts of Interest}

The authors declare that there is no conflict of interest regarding the publication of this article.

\section{References}

[1] S.-K. Yang, W. Chen, C.-H. Su, and C.-H. Liu, "Incidence and comorbidity of dementia with Lewy bodies: a populationbased cohort study," Behavioural Neurology, vol. 2018, Article ID 7631951, 6 pages, 2018.

[2] D. H. Hepp, D. L. E. Vergoossen, E. Huisman et al., "Distribution and load of amyloid- $\beta$ pathology in Parkinson disease and dementia with Lewy bodies," Journal of Neuropathology \& Experimental Neurology, vol. 75, no. 10, pp. 936-945, 2016.

[3] J. E. Ware Jr and C. D. Sherbourne, "The MOS 36-item shortform health survey (SF-36): I. Conceptual framework and item selection," Medical Care, vol. 30, no. 6, pp. 473-483, 1992.

[4] H.-M. Chen and C.-M. Chen, "Factors associated with quality of life among older adults with chronic disease in Taiwan," International Journal of Gerontology, vol. 11, no. 1, pp. 1215, 2017. 
[5] S. Banerjee, K. Samsi, C. D. Petrie et al., "What do we know about quality of life in dementia? A review of the emerging evidence on the predictive and explanatory value of disease specific measures of health related quality of life in people with dementia," International Journal of Geriatric Psychiatry, vol. 24, no. 1, pp. 15-24, 2009.

[6] C. Mueller, C. Ballard, A. Corbett, and D. Aarsland, "The prognosis of dementia with Lewy bodies," Lancet Neurology, vol. 16, no. 5, pp. 390-398, 2017.

[7] P. Thomas, F. Lalloué, P. M. Preux et al., "Dementia patients caregivers quality of life: the PIXEL study," International Journal of Geriatric Psychiatry, vol. 21, no. 1, pp. 50-56, 2006.

[8] R. Figari-Jordan, K. Anderson, A. Gruber-Baldini et al., "Comparison of quality of life and disability in three different dementias (P07.180)," Neurology, vol. 78, no. Meeting Abstracts 1, article P07.180, 2012.

[9] F. Boström, L. Jönsson, L. Minthon, and E. Londos, "Patients with dementia with lewy bodies have more impaired quality of life than patients with Alzheimer disease," Alzheimer Disease \& Associated Disorders, vol. 21, no. 2, pp. 150-154, 2007.

[10] L. P. Sands, P. Ferreira, A. L. Stewart, M. Brod, and K. Yaffe, "What explains differences between dementia patients' and their caregivers' ratings of patients' quality of life?," The American Journal of Geriatric Psychiatry, vol. 12, no. 3, pp. 272-280, 2004.

[11] J. H. T. Karlawish, D. Casarett, J. Klocinski, and C. M. Clark, "The relationship between caregivers' global ratings of Alzheimer's disease patients' quality of life, disease severity, and the caregiving experience," Journal of the American Geriatrics Society, vol. 49, no. 8, pp. 1066-1070, 2001.

[12] F. Yang, P. Dawes, I. Leroi, and B. Gannon, "Measurement tools of resource use and quality of life in clinical trials for dementia or cognitive impairment interventions: a systematically conducted narrative review," International Journal of Geriatric Psychiatry, vol. 33, no. 2, pp. e166-e176, 2018.

[13] R. G. Logsdon, L. E. Gibbons, S. M. McCurry, and L. Teri, "Quality of life in Alzheimer's disease: patient and caregiver reports," Journal of Mental health and Aging, vol. 5, pp. 2132, 1999 .

[14] S. Smith, D. Lamping, S. Banerjee et al., "Measurement of health-related quality of life for people with dementia: development of a new instrument (DEMQOL) and an evaluation of current methodology," Health Technology Assessment, vol. 9, no. 10, 2005 .

[15] The EuroQol Group, "EuroQol - a new facility for the measurement of health-related quality of life," Health Policy, vol. 16, no. 3, pp. 199-208, 1990.

[16] C. Jenkinson, R. Layte, D. Jenkinson et al., "A shorter form health survey: can the SF-12 replicate results from the SF-36 in longitudinal studies?," Journal of Public Health, vol. 19, no. 2, pp. 179-186, 1997.

[17] A. A. Mougias, A. Politis, C. G. Lyketsos, and V. G. Mavreas, "Quality of life in dementia patients in Athens, Greece: predictive factors and the role of caregiver-related factors," International Psychogeriatrics, vol. 23, no. 3, pp. 395-403, 2011.

[18] C. Ballard, C. Holmes, I. McKeith et al., "Psychiatric morbidity in dementia with Lewy bodies: a prospective clinical and neuropathological comparative study with Alzheimer's disease," American Journal of Psychiatry, vol. 156, no. 7, pp. 10391045, 1999.
[19] T. J. Ferman, G. E. Smith, B. F. Boeve et al., "Neuropsychological differentiation of dementia with Lewy bodies from normal aging and Alzheimer's disease," The Clinical Neuropsychologist, vol. 20, no. 4, pp. 623-636, 2006.

[20] T. J. Ferman, Z. Arvanitakis, H. Fujishiro et al., "Pathology and temporal onset of visual hallucinations, misperceptions and family misidentification distinguishes dementia with Lewy bodies from Alzheimer's disease," Parkinsonism \& Related Disorders, vol. 19, no. 2, pp. 227-231, 2013.

[21] P. Thaipisuttikul, I. Lobach, Y. Zweig, A. Gurnani, and J. E. Galvin, "Capgras syndrome in dementia with Lewy bodies," International Psychogeriatrics, vol. 25, no. 5, pp. 843-849, 2013.

[22] D. Erskine, A. J. Thomas, J. P. Taylor et al., "Neuronal loss and A-synuclein pathology in the superior colliculus and its relationship to visual hallucinations in dementia with Lewy bodies," The American Journal of Geriatric Psychiatry, vol. 25, no. 6, pp. 595-604, 2017.

[23] S. López-Pousa, J. Vilalta-Franch, J. Garre-Olmo, S. Pons, and M. G. Cucurella, "Characterisation and prevalence of the psychological and behavioural symptoms in patients with dementia," Revista de Neurologia, vol. 45, no. 11, pp. 683-688, 2007.

[24] R.-C. Tzeng, C.-F. Tsai, C.-T. Wang, T.-Y. Wang, and P.Y. Chiu, "Delusions in patients with dementia with Lewy bodies and the associated factors," Behavioural Neurology, vol. 2018, Article ID 6707291, 8 pages, 2018.

[25] U. P. Mosimann, E. N. Rowan, C. E. Partington et al., "Characteristics of visual hallucinations in Parkinson disease dementia and dementia with Lewy bodies," The American Journal of Geriatric Psychiatry, vol. 14, no. 2, pp. 153-160, 2006.

[26] J. Cummings, S. Isaacson, R. Mills et al., "Pimavanserin for patients with Parkinson's disease psychosis: a randomised, placebo-controlled phase 3 trial," The Lancet, vol. 383, no. 9916, pp. 533-540, 2014.

[27] M. Baquero and N. Martin, "Depressive symptoms in neurodegenerative diseases," World Journal of Clinical Cases, vol. 3, no. 8, pp. 682-693, 2015.

[28] K. Ornstein, J. E. Gaugler, D. P. Devanand, N. Scarmeas, C. Zhu, and Y. Stern, "The differential impact of unique behavioral and psychological symptoms for the dementia caregiver: how and why do patients' individual symptom clusters impact caregiver depressive symptoms?," The American Journal of Geriatric Psychiatry, vol. 21, no. 12, pp. 1277-1286, 2013.

[29] Y. Yamane, K. Sakai, and K. Maeda, "Dementia with Lewy bodies is associated with higher scores on the Geriatric Depression Scale than is Alzheimer's disease," Psychogeriatrics, vol. 11, no. 3, pp. 157-165, 2011.

[30] K. Kurisu, S. Terada, E. Oshima et al., "Comparison of QOL between patients with different degenerative dementias, focusing especially on positive and negative affect," International Psychogeriatrics, vol. 28, no. 8, pp. 1355-1361, 2016.

[31] J. A. Pillai and J. B. Leverenz, "Sleep and neurodegeneration: a critical appraisal," Chest, vol. 151, no. 6, pp. 1375-1386, 2017.

[32] C. Peyron, D. K. Tighe, A. N. van den Pol et al., "Neurons containing hypocretin (orexin) project to multiple neuronal systems," Journal of Neuroscience, vol. 18, no. 23, pp. $9996-$ 10015, 1998.

[33] M. Wennström, E. Londos, L. Minthon, and H. M. Nielsen, "Altered CSF orexin and a-synuclein levels in dementia patients," Journal of Alzheimer's Disease, vol. 29, no. 1, pp. 125-132, 2012. 
[34] T. J. Ferman, B. F. Boeve, G. E. Smith et al., "Inclusion of RBD improves the diagnostic classification of dementia with Lewy bodies," Neurology, vol. 77, no. 9, pp. 875-882, 2011.

[35] W. C. Pao, B. F. Boeve, T. J. Ferman et al., "Polysomnographic findings in dementia with Lewy bodies," The Neurologist, vol. 19, no. 1, pp. 1-6, 2013.

[36] L. Allan, I. McKeith, C. Ballard, and R. A. Kenny, "The prevalence of autonomic symptoms in dementia and their association with physical activity, activities of daily living and quality of life," Dementia and Geriatric Cognitive Disorders, vol. 22 , no. 3, pp. 230-237, 2006.

[37] G. E. Berrios, C. Campbell, and B. E. Politynska, "Autonomic failure, depression and anxiety in Parkinson's disease," British Journal of Psychiatry, vol. 166, no. 6, pp. 789-792, 1995.

[38] C. O. L. L. E. E. N. A. MCHORNEY, W. JOHNE JR., and R. ANASTASIAE, "The MOS 36-Item Short-Form Health Survey (SF-36): II. Psychometric and clinical tests of validity in measuring physical and mental health constructs," Medical Care, vol. 31, no. 3, pp. 247-263, 1993.

[39] A. Fanciulli, S. Strano, C. Colosimo, C. Caltagirone, G. Spalletta, and F. E. Pontieri, "The potential prognostic role of cardiovascular autonomic failure in $\alpha$-synucleinopathies," European Journal of Neurology, vol. 20, no. 2, pp. 231-235, 2013.

[40] H. Oka, M. Morita, K. Onouchi, M. Yoshioka, S. Mochio, and K. Inoue, "Cardiovascular autonomic dysfunction in dementia with Lewy bodies and Parkinson's disease," Journal of the Neurological Sciences, vol. 254, no. 1-2, pp. 72-77, 2007.

[41] H. Oka, M. Yoshioka, M. Morita et al., "Reduced cardiac 123I-MIBG uptake reflects cardiac sympathetic dysfunction in Lewy body disease," Neurology, vol. 69, no. 14, pp. 1460-1465, 2007.

[42] Y. Horimoto, M. Matsumoto, H. Akatsu et al., "Autonomic dysfunctions in dementia with Lewy bodies," Journal of Neurology, vol. 250, no. 5, pp. 530-533, 2003.

[43] P. Thaisetthawatkul, B. F. Boeve, E. E. Benarroch et al., "Autonomic dysfunction in dementia with Lewy bodies," Neurology, vol. 62, no. 10, pp. 1804-1809, 2004.

[44] R. Freeman, W. Wieling, F. B. Axelrod et al., "Consensus statement on the definition of orthostatic hypotension, neurally mediated syncope and the postural tachycardia syndrome," Autonomic Neuroscience, vol. 161, no. 1-2, pp. 46-48, 2011.

[45] B. P. Boot, "Comprehensive treatment of dementia with Lewy bodies," Alzheimer's Research \& Therapy, vol. 7, no. 1, p. 45, 2015.

[46] R. Kenny, F. E. Shaw, J. T. O'Brien, P. H. Scheltens, R. Kalaria, and C. Ballard, "Carotid sinus syndrome is common in dementia with Lewy bodies and correlates with deep white matter lesions," Journal of Neurology, Neurosurgery \& Psychiatry, vol. 75, no. 7, pp. 966-971, 2004.

[47] K. Stubendorff, O. Hansson, L. Minthon, and E. Londos, "Differences in survival between patients with dementia with Lewy bodies and patients with Alzheimer's disease - measured from a fixed cognitive level," Dementia and Geriatric Cognitive Disorders, vol. 32, no. 6, pp. 408-416, 2012.

[48] M. Darwish, M. Kirby, E. T. Hellriegel, and P. Robertson Jr, "Armodafinil and modafinil have substantially different pharmacokinetic profiles despite having the same terminal half-lives," Clinical Drug Investigation, vol. 29, no. 9, pp. 613-623, 2009.
[49] M. I. Lapid, K. M. Kuntz, S. S. Mason et al., "Efficacy, safety, and tolerability of armodafinil therapy for hypersomnia associated with dementia with Lewy bodies: a pilot study," Dementia and Geriatric Cognitive Disorders, vol. 43, no. 5-6, pp. 269280, 2017.

[50] V. Larsson, K. Engedal, D. Aarsland, C. Wattmo, L. Minthon, and E. Londos, "Quality of life and the effect of memantine in dementia with Lewy bodies and Parkinson's disease dementia," Dementia and Geriatric Cognitive Disorders, vol. 32, no. 4, pp. 227-234, 2011.

[51] M. Inskip, Y. Mavros, P. S. Sachdev, and M. A. Fiatarone Singh, "Exercise for individuals with Lewy body dementia: a systematic review," PLoS One, vol. 11, no. 6, article e0156520, 2016.

[52] L. Bonanni, A. Cagnin, F. Agosta et al., "The Italian dementia with Lewy bodies study group (DLB-SINdem): toward a standardization of clinical procedures and multicenter cohort studies design," Neurological Sciences, vol. 38, no. 1, pp. 8391, 2017. 


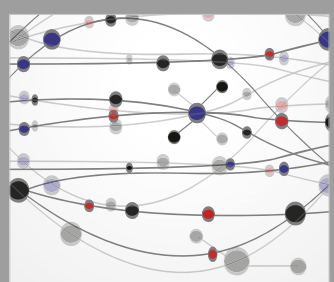

The Scientific World Journal
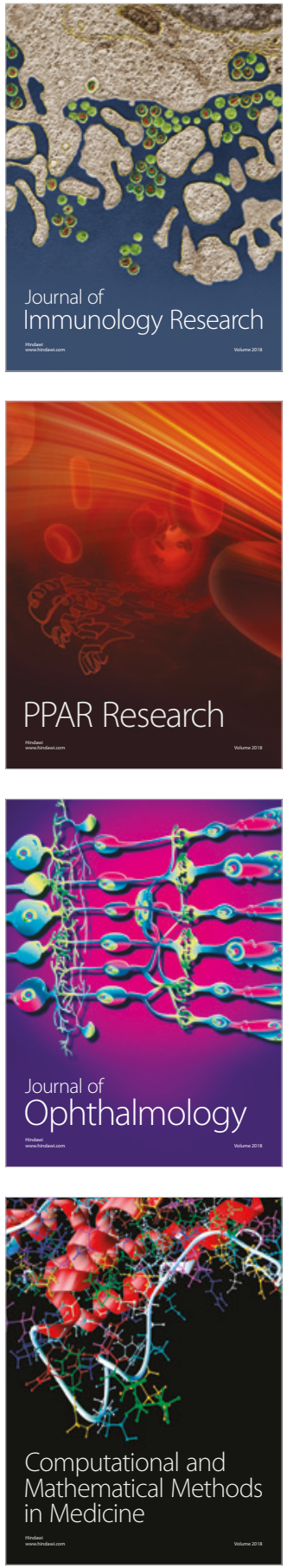

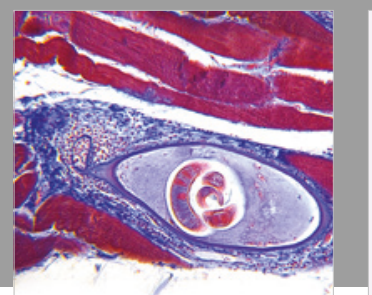

Gastroenterology Research and Practice

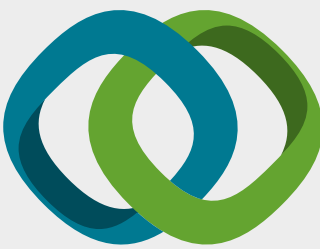

\section{Hindawi}

Submit your manuscripts at

www.hindawi.com
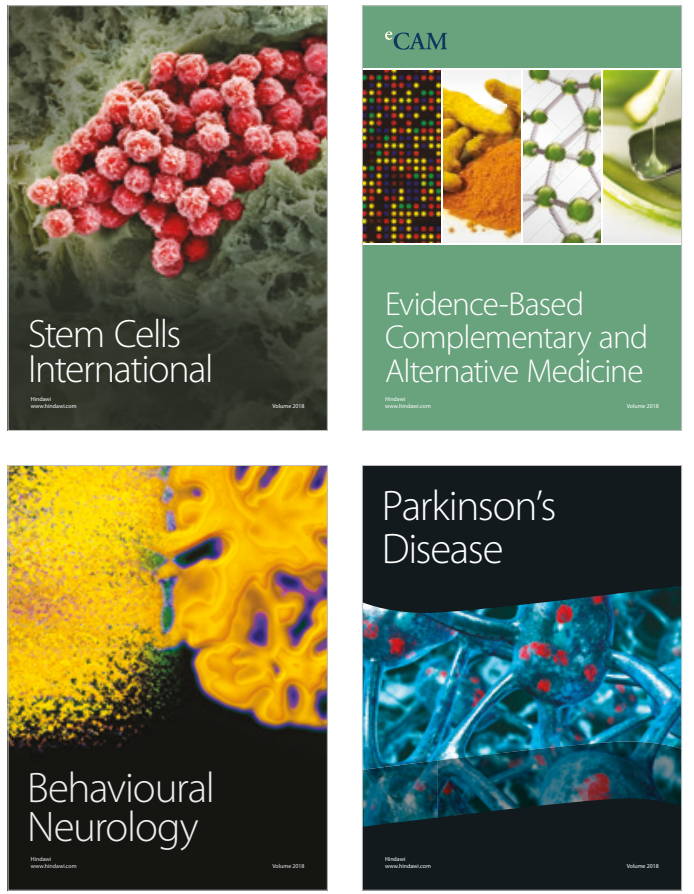

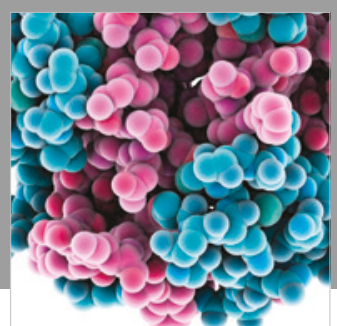

ournal of

Diabetes Research

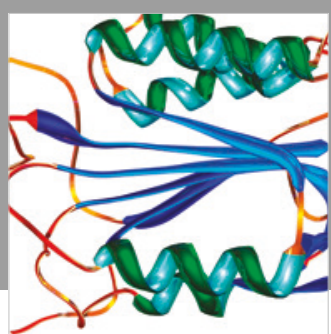

Disease Markers
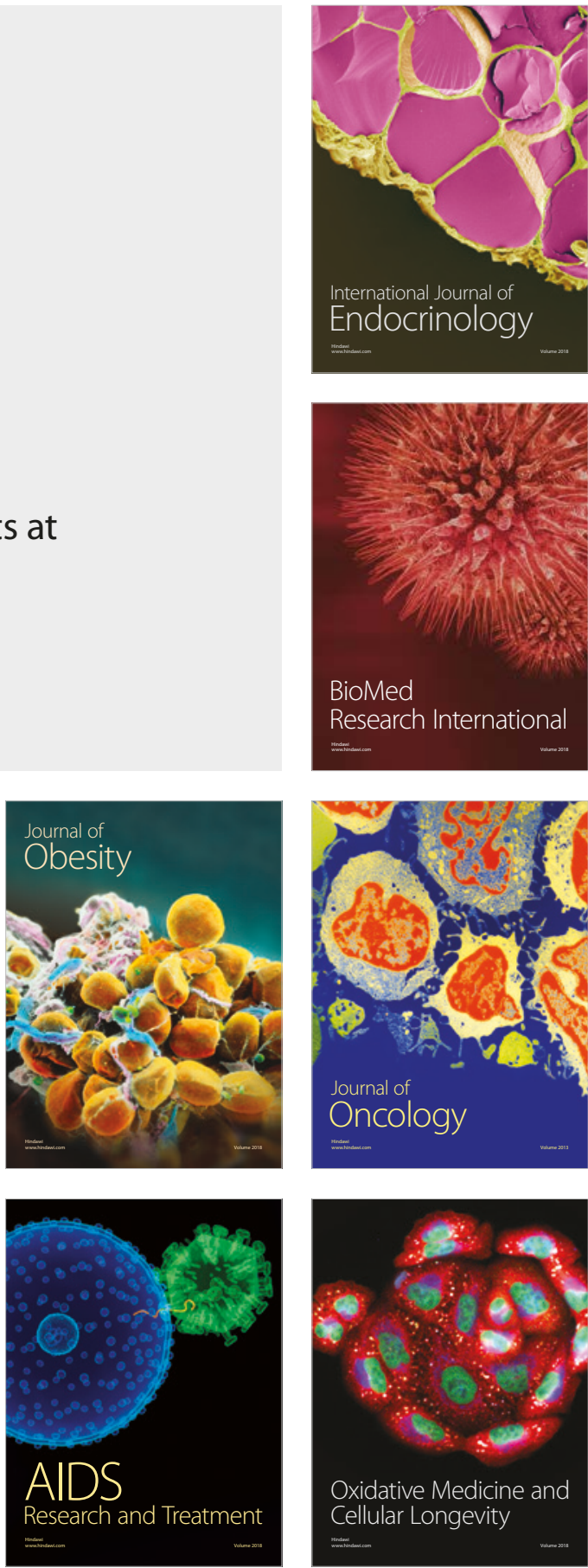